\title{
Comunicación
}

\section{Rehabilitación y mantenimiento en cautiverio de ranas altoandinas (Telmatobius spp) decomisadas en la ciudad de Lima, Perú}

\author{
Rehabilitation and maintenance in captivity of High Andean frogs (Telmatobius \\ spp) confiscated in Lima, Peru
}

\author{
Roberto Elías Piperis ${ }^{1,3,4}$, Raúl Berenguel Cook$^{2}$, Erick Reátegui Guzmán ${ }^{2}$, \\ Thomas J. Weaver ${ }^{3}$
}

\section{Resumen}

Las ranas del género Telmatobius están entre las especies de fauna silvestre más traficadas en Perú debido a que son colectadas para ser usadas como fuente alimenticia o porque les atribuyen propiedades medicinales. Entre 2012 y 2013, el Laboratorio de Vida Silvestre de la Facultad de Medicina Veterinaria y Zootecnia de la Universidad Peruana Cayetano Heredia recibió y mantuvo cerca de 400 ranas altoandinas (Telmatobius spp) de decomisos hechos en el Mercado de La Parada en La Victoria, Lima, por la Administración Técnica Forestal y de Fauna Silvestre de Lima. En este estudio se recopila información producto del trabajo en la rehabilitación y mantenimiento en cautiverio de estos ejemplares.

Palabras clave: Telmatobius, tráfico, altoandino, amenazado

\footnotetext{
${ }^{1}$ Laboratorio de Vida Silvestre, Facultad de Medicina Veterinaria y Zootecnia, Universidad Peruana Cayetano Heredia

${ }^{2}$ Facultad de Medicina Veterinaria y Zootecnia, Universidad Peruana Cayetano Heredia, Lima, Perú

${ }^{3}$ Denver Zoological Foundation, Colorado, United States of America

${ }^{4}$ E-mail: roberto.elias@upch.pe
}

Recibido: 26 de marzo de 2021

Aceptado para publicación: 7 de octubre de 2021

Publicado: 22 de diciembre de 2021

CLos autores. Este artículo es publicado por la Rev Inv Vet Perú de la Facultad de Medicina Veterinaria, Universidad Nacional Mayor de San Marcos. Este es un artículo de acceso abierto, distribuido bajo los términos de la licencia Creative Commons Atribución 4.0 Internacional (CC BY 4.0) [https:// creativecommons.org/licenses/by/4.0/deed.es] que permite el uso, distribución y reproducción en cualquier medio, siempre que la obra original sea debidamente citada de su fuente original 
Frogs of the genus Telmatobius are among the most trafficked wildlife species in Peru because they are captured as a source of food or because they are attributed medicinal properties. Between 2012 and 2013, the Wildlife Laboratory of the Facultad de Medicina Veterinaria y Zootecnia de la Universidad Peruana Cayetano Heredia received and kept close to 400 High Andean frogs (Telmatobius spp) from confiscation made at La Parada Market in La Victoria, Lima by the Technical Forestry and Wildlife Administration of Lima. In this study, information compiled as a result of work done in the rehabilitation and maintenance of these specimens in captivity is provided.

Key words: Telmatobius, traffic, high Andean, threatened

\section{INTRODUCCIÓN}

El género Telmatobius es un grupo diverso de especies de anfibios que habita los Andes desde Ecuador hasta Chile y Argentina entre los 1000 a $5200 \mathrm{msnm}$ (Vellard, 1951). Este género incluye 63 especies (AmphibiaWeb, 2021) de las cuales 55 $(\mathrm{H} » 87 \%)$ se encuentran categorizadas como amenazadas por la Lista Roja de la Unión Internacional para la Conservación de la Naturaleza (The IUCN Red List of Threatened Species, 2021).

En el Perú existen 28 especies de este género, donde 17 (alrededor del 60\%) han sido categorizadas como amenazadas (en peligro crítico, en peligro o en situación vulnerable) por el Estado Peruano (D.S. 0042014-MINAGRI) (SERFOR, 2018a). Las amenazas que se han identificado para los anfibios andinos en el Perú incluyen la pérdida de hábitat, enfermedades infecciosas emergentes como la quitridiomicosis, degradación del hábitat, la introducción de especies exóticas invasoras y la sobrexplotación como alimento para consumo humano, que probablemente esté afectando a 13 especies (Angulo 2008; Aguilar et al., 2010). Estos anfibios se encuentran entre las especies de fauna silvestre más traficadas, especialmente T. culeus (SERFOR, 2017). Su captura es con fines de autoconsumo y comercio por ser una fuente de proteína y porque se le atribuyen propiedades medicinales (Angulo, 2008).

En 2008, la Facultad de Medicina Veterinaria y Zootecnia de la Universidad Peruana Cayetano Heredia, con ayuda de la Fundación Zoológica de Denver, creó el Laboratorio de Vida Silvestre con el objetivo generar información sobre rehabilitación y mantenimiento de ranas del género Telmatobius en cautiverio a partir de ejemplares recuperados del tráfico.

\section{Materiales y MéTodos}

El estudio recopila información del trabajo de rehabilitación y mantenimiento del segundo grupo y más numeroso de ranas altoandinas (Telmatobius spp) recibidas hasta el 2013 por el Laboratorio de Vida Silvestre (LVS) de la Facultad de Medicina Veterinaria y Zootecnia de la Universidad Peruana Cayetano Heredia (FAVEZ-UPCH), ubicado en el distrito de San Martín de Porres, provincia y departamento de Lima, Perú, para su evaluación sanitaria, rehabilitación y mantenimiento en cautiverio. Las ranas fueron decomisadas en el Mercado de La Parada del distrito de La Victoria en Lima, en enero del 2012, por la Administración Técnica Fo- 


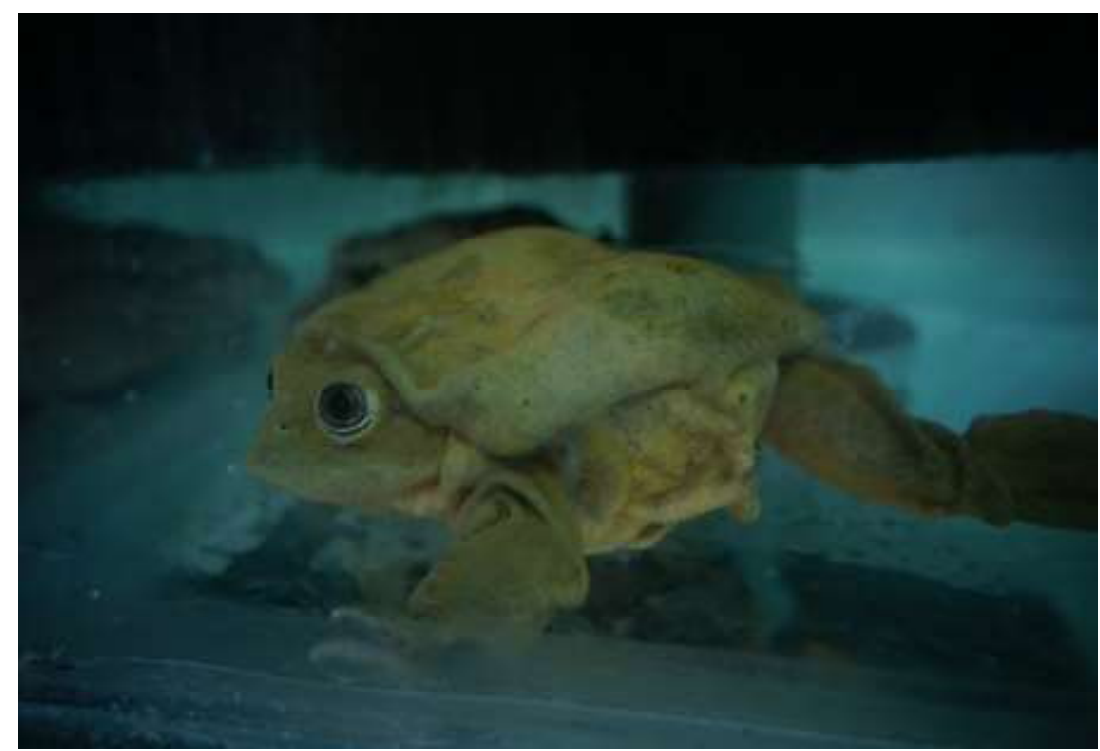

Figura 1. Ejemplar de rana del Titicaca (Telmatobius culeus) en proceso de rehabilitación en el Laboratorio de Vida Silvestre de la Facultad de Medicina Veterinaria y Zootecnia de la Universidad Peruana Cayetano Heredia (Lima, Perú)

restal y de Fauna Silvestre de Lima (ATFFS Lima). Esto se realizó como parte del proyecto de conservación de la rana del Titicaca (Telmatobius culeus; Figura 1), en alianza entre FAVEZ-UPCH, el Parque Zoológico Huachipa (PZH) y la Fundación Zoológica de Denver (FZD), donde el LVS fue registrado por el PZH ante la ATFFS Lima, como el área responsable de la cuarentena de las ranas que pasarían a ser parte de la colección de ese centro de cría, y donde se exhibirían con fines educativos.

Este proyecto se inició en el 2008, incluyendo actividades de educación ambiental, investigación y rehabilitación y exhibición de estos animales con fines educativos en ambos zoológicos (PZH y FDZ). El LVS recibió y mantuvo ejemplares desde 2008 hasta 2017 , siendo este el lote más numeroso que se intentó rehabilitar y mantener hasta su traslado al PZH. Previo a esta experien- cia, hubo un grupo reducido de 10 animales y después del 2013 se recibieron grupos pequeños de 30 individuos como máximo.

El proceso de cuarentena en el LVS se iniciaba con el lavado, desinfección y preparación de los ambientes (acuarios o cajas plásticas), recepción de los individuos, evaluación sanitaria y tratamiento para la rehabilitación de los animales, y finalmente el mantenimiento de estos hasta su traslado al PZH. La duración del periodo de cuarentena fue de $90 \mathrm{~d}$ y el tiempo de su mantenimiento en el laboratorio luego de este periodo dependía de la disponibilidad de espacio en el zoológico. La evaluación sanitaria se realizaba semanalmente, empezando en el día 1, y estaba comprendida por evaluaciones de parámetros físicosquímicos del agua, exámenes físicos de los animales y, si en caso fuera necesario, exámenes coproparasitológicos y microbiológicos con el tratamiento veterinario respectivo. 


\section{Resultados}

En enero de 2012, aproximadamente 400 ranas altoandinas adultas del género Telmatobius fueron derivadas al LVS por la ATFFS Lima. A1 llegar los individuos fueron separados según su estado de salud y distribuidos equitativamente (aproximadamente 40 individuos por ambiente) en cinco cajas plásticas de $120 \mathrm{~L}$ de capacidad y en cinco acuarios de vidrio de $100 \times 30 \mathrm{~cm}$ y $46 \mathrm{~cm}$ de alto (previo a esto solo se habían mantenido dos ranas por acuario), con agua potable previamente tratada con Ammo Buster ${ }^{\mathrm{TM}}$ Extreme para eliminar el cloro y neutralizar nitritos. Cada ambiente contaba con un filtro biológico de esponja con bomba, el cual era limpiado cada $10-14 \mathrm{~d}$.

Las cajas y acuarios eran mantenidos en una habitación cerrada con aire acondicionado que permitía mantener en $17^{\circ} \mathrm{C}$ la temperatura ambiental; además, uno de los acuarios estaba conectado a un enfriador de agua Boyu ${ }^{\mathrm{TM}} 800-2200 \mathrm{~L} / \mathrm{h}$ (Modelo L-200) que permitía bajar la temperatura hasta $13^{\circ} \mathrm{C}$. Dos tercios del agua era cambiada cada 7$10 \mathrm{~d}$ y cada $30-45 \mathrm{~d}$ eran lavados con agua y detergente. Los componentes químicos del agua fueron evaluados usando un kit de prueba básica para acuarios Sera ${ }^{\mathrm{TM}}$ Aqua-Test Set. Los valores promedio durante esta etapa fueron de $7.5 \mathrm{de} \mathrm{pH}, 1 \mathrm{ppm}$ de nitritos, $40 \mathrm{mg} / 1$ de nitratos y 0 ppm de amoniaco.

En los acuarios se colocaron plantas decorativas de plástico, grava y piedras redondeadas de río para que sean utilizados por las ranas como refugio y posadero. La alimentación era interdiaria y se basó en comida viva. Al inició se utilizó Tubifex tubifex y se fue complementado con otros insumos como Poecilia reticulata y $P$. sphenops, hasta llegar acostumbrarlos a alimentos comerciales como Tropical ${ }^{\mathrm{TM}}$ Carnivore.

La mayoría de estas ranas se encontraba en mala condición corporal, con anorexia y letargia, y fueron muriendo dentro de las dos a tres primeras semanas de recibidas. Finalmente sobrevivieron 14 individuos en las cinco peceras. En los animales que murieron se observaron lesiones como eritema, hemorragias y erosiones cutáneas compatible con dermatoseptisemia bacteriana, así como abundantes nematodos del género Hedruris en el tracto intestinal. Se tomaron hisopados cutáneos de algunos animales $(n=6)$ para microbiología que fueron analizadas en el Laboratorio de Microbiología de FAVEZ-UPCH, donde se aislaron bacterias del género Aeromonas y Pseudomonas en cuatro muestras, y dos dieron positivo a Saprolegnia. Estos últimos individuos presentaban lesiones típicas («algodones») de este tipo de infecciones micóticas.

Además, se tomaron muestras de 62 ranas para el diagnóstico de Batrachochytrium dendrobatidis $(\mathrm{Bd})$ para su análisis en el laboratorio Pisces Molecular LLC, USA, resultando $60 \%(n=37)$ positivos a ese patógeno (Zevallos et al., 2016). Dentro del periodo de cuarentena, los animales sobrevivientes $(n=14)$ fueron tratados contra $B d$ con baños con itraconazol al $0.01 \%$ en agua por 5 min cada 24 h durante $11 \mathrm{~d}$ (Carpenter 2005) y se les administró dos veces fenbendazol (50 $\mathrm{mg} / \mathrm{kg} \mathrm{VO}$ ) cada $15 \mathrm{~d}$ para reducir la carga parasitaria (Carpenter, 2005) Las ranas sobrevivientes no presentaron otro problema sanitario durante el 2012 al 2013 que permanecieron en el LVS, en el que no se recibieron otros ejemplares.

\section{Discusión}

Las ranas altoandinas están dentro del grupo de especies de fauna silvestre más traficadas del Perú, siendo la rana del Titicaca la que más se captura (SERFOR, 2017). En un decomiso se pueden recuperar hasta 2500 individuos (W. Silva, comunicación personal, 2019), pero lamentablemente no existe información sobre rehabilitación y mantenimiento en cautiverio de estas especies, y menos sobre centros de cría dedicados a este tema. El 
presente estudio pretende compartir información obtenida en el LVS, durante los años 2012 y 2013, cuando se recibió un grupo (cerca de 400) de estos anfibios para su rehabilitación y mantenimiento.

Las ranas del género Telmatobius son especies de hábitos acuáticos o semi acuáticos, de allí que mantenerlas en cautiverio es muy similar a la crianza de peces ornamentales. Se debe tener un acuario o recipiente que pueda mantener volúmenes grandes de agua y sea fácil de limpiar. Como el agua es el medio en donde ellas viven, se alimentan y eliminan sus deposiciones, debe estar constantemente evaluada, lo cual se puede hacer con un kit de pruebas básicas para acuarios, que permite determinar la frecuencia de renovación del agua y de limpieza de los ambientes, lo cual depende de la biomasa y el volumen de agua. Se debe considerar que ambientes sobrepoblados pueden afectar directamente en los parámetros químicos del agua, en la competencia por el alimento y en la transmisión de enfermedades.

En las evaluaciones del agua se encontraron valores de $\mathrm{pH}$ ligeramente por debajo al que se encuentra en su hábitat natural que suele ser de 8 (Genova, 2011), pero por encima de 7 que es aceptable para anfibios. Un valor ácido puede alterar el balance iónico fisiológico y afectar su supervivencia (Beattie y Tyler-Jones, 1992). Se recomienda que los niveles de nitritos sean de $0 \mathrm{ppm}$ porque son elementos tóxicos; sin embargo, en el LVS se encontraron valores superiores, al igual que los niveles de nitrato. La acumulación de ambos es un indicador de cambio de agua insuficiente (Odum y Zippel, 2011), que en este caso estaba relacionado a la gran cantidad de animales que se mantuvieron por ambiente, ya que se pasó de mantener dos animales por recinto a aproximadamente 40 . El tener estos valores elevados pueden conllevar a metaglobinemia, lo que puede comprometer el transporte de oxígeno a los tejidos (Diana et al., 2001). Los anfibios poseen una piel permeable que les permite respirar a través de ella e intercambiar sustancias con su entorno, actuando como órgano de respiración y para mantener el equilibrio hídrico y electrolítico (Duellman y Trueb, 1986), lo que los hace más susceptibles que otros taxones a sustancias extrañas en su medio.

Otra característica de estos vertebrados es de ser ectotermos; es decir, que regulan su temperatura corporal con ayuda de la temperatura ambiental (Pough, 1980), de allí que sea necesario mantener la temperatura ambiental cercana a la de su hábitat natural, lo cual se logró con el aire acondicionado y el enfriador de agua instalados en LVS.

La alimentación es otro punto importante en el mantenimiento de anfibios. Los adultos se alimentan de presas (animales vivos), por eso es clave asegurar la comida, sea produciéndola o asegurando un proveedor, antes de recibir a las ranas. El uso de alimento comercial es una opción, pero solo se puede ir introduciendo en forma paulatina.

Factores externos, como temperatura incorrecta, agua sucia o falta de alimento que les cause estrés puede predisponerlos a enfermarse. Es común que desarrollen infecciones a partir de bacterias que conforman la flora normal de su piel. El aislamiento de Aeromonas y Pseudomonas en ranas es muy común ya que estas suelen ser parte de la flora normal (Edery, 2015), pero suelen convertirse en patógenos oportunistas cuando el individuo se encuentra inmunodeprimido. En el caso de los individuos decomisados era de esperarse que desarrollen infecciones bacterianas porque pasaron por un proceso muy estresante de varios días, desde captura en su hábitat, acopio realizado por el traficante hasta su transporte en cajas de madera a la ciudad de Lima (SERFOR, 2017). En todo ese tiempo, permanecieron hacinadas a temperatura ambiental, con poca agua y sin alimentación. Muchas mueren en este lapso con infecciones septicémicas conocidas como la «enfermedad de la pierna roja», causada por Aeromonas u otras bacterias que son parte de su flora (Hird et al., 1981). Otra enfermedad común en estos casos es la 
saprolegniosis, que es producida por un hongo oportunista cuando los animales pierden integridad del tegumento por lesiones físicas (Kim et al., 2008).

Un problema alarmante que ocasiona el tráfico de anfibios es la transmisión y diseminación de enfermedades infecciosas emergentes, como la quitridiomicosis. Esta enfermedad es muy contagiosa, de fácil transmisión y ya ha sido reportada en Telmatobius en un mercado de Cusco (Catenazzi et al., 2010) y en muestras analizadas en el LVS (Zevallos et al., 2016). Los animales positivos a esta micosis no deben ser reunidos con otros anfibios hasta que pasen por un tratamiento que puede ser con itraconazol y, en lo posible, pasen por una segunda evaluación para confirmar la efectividad del tratamiento. Tampoco deben ser liberadas en áreas naturales como ha sucedido en algunas ocasiones (SERFOR 2018b), ya que se trata de una enfermedad letal que ha extinguido casi 100 especies de anfibios e infectado a casi 700 en todos los continentes donde estas habitan (Lips, 2016).

Para la rehabilitación y el mantenimiento de ranas altoandinas (Telmatobius spp) en cautiverio se necesita contar con conocimientos sobre manejo de acuarios y cría de peces ornamentales porque estos grupos tienen requerimientos similares. Asimismo, el tráfico de ranas puede incrementar la presentación de enfermedades infecciosas entre estos individuos, por lo cual se debe tener mucho cuidado con el manejo y disposición que se haga con estos animales.

\section{Literatura Citada}

1. Aguilar C, Ramírez C, Rivera D, SiuTing K, Suarez J, Torres C. 2010. Anfibios andinos del Perú fuera de Áreas Naturales Protegidas: amenazas y estado de conservación. Rev Peru Biol 17: 5-28.
2. Angulo A. 2008. Conservation needs of Batrachophrynus and Telmatobius frogs of the Andes of Peru. Conservat Soc 6: 628-633.

3. Beattie RC, Tyler-Jones R. 1992. The effects of low $\mathrm{pH}$ and aluminum on breeding success in the frog Rana temporaria. J Herpetol 26: 353-360.

4. AmphibiaWeb. 2021. Search Database. [Internet]. Disponible en: https:// amphibiaweb.org/search/index.html

5. Carpenter JW. 2005. Exotic animal formulary. $3^{\text {rd }}$ ed. USA: Elsevier Saunders. 496 p.

6. Catenazzi A, Vredenburg VT, Lehr E. 2010. Batrachochytrium dendrobatidis in the live frog trade of Telmatobius (Anura: Ceratophryidae) in the tropical Andes. Dis Aquat Organ 92: 187-191. doi: 10.3354/dao02250

7. Diana SG, Beasley VB, Wright KM. 2001. Clinical toxicology. In: Wright KM, Whitaker BR (eds). Amphibian medicine and captive husbandry. Malabar FL: Krieger Publishing. p 223-232.

8. Duellman WE, Trueb L. 1986. Biology of amphibians. London: John Hopkins University Press. 620 p.

9. Edery S. 2015. Bacterias aisladas de la piel de la rana gigante del Titicaca (Telmatobius culeus) en condiciones de cautiverio. Tesis de Médico Veterinario. Lima: Univ. Peruana Cayetano Heredia. 19 p.

10. Genova M. 2011. Density and habitat preferences of Lake Titicaca frog (Telmatobius culeus) at North-West of Copacabana peninsula. MSci Thesis. The Netherlands: Wageningen Univer-sity.

11. Hird DW, Diesch SL, McKinnell, Gorham E, Martin FB, Kurtz SW, Dubrovolny C. 1981. Aeromonas hydrophila in wild-caught frogs and tadpoles (Rana pipiens) in Minnesota. Lab Anim Sci 31: 166-169.

12. Kim S, Eom AH, Park D, Ra NY. 2008. Detection of infectious fungal diseases of frogs inhabiting in Korea. 
Mycobiology 36: 10-12. doi: 10.4489/ MYCO.2008.36.1.010

13. Lips KR. 2016. Overview of chytrid emergence and impacts on amphibians. Philos T Roy Soc B 371: 20150465. doi: 10.1098/rstb.2015.0465

14. Odum RA, Zippel K. 2011. Water quality. [Internet]. Available in: http:// www.amphibianark.org/wp-content/ uploads/2018/08/Water-Quality-OdumZippel-2011.pdf

15. Pough F. 1980. The advantages of ectothermy for tetrapods. Am Nat 115: 92-112.

16. [SERFOR] Servicio Nacional Forestal y de Fauna Silvestre. 2017. Estrategia nacional para reducir el tráfico ilegal de fauna silvestre en el Perú 2017-2027. SERFOR. [Internet]. Disponible: https:// www.serfor.gob.pe/portal/estrategia-nacional-para-reducir-el-trafico-ilegal-defauna-silvestre-al-2027-en-el-peru

17. [SERFOR] Servicio Nacional Forestal y de Fauna Silvestre. 2018a. Libro rojo de la fauna silvestre amenazada del Perú. Servicio Nacional Forestal y de Fauna Silvestre. [Internet]. Disponible en: https://www.serfor.gob.pe/portal/wpcontent/uploads/2018/10/Libro-Rojo.pdf

18. [SERFOR] Servicio Nacional Forestal y de Fauna Silvestre. $2018 b$. SERFOR rescata y libera más de 1,900 ranas que eran transportadas ilegalmente. Servicio Nacional Forestal y de Fauna Silvestre. [Internet]. Disponible: https://www.serfor.gob.pe/portal/noticias/serfor-rescata-y-libera-mas-de1900-ranas-que-eran-transportadas-ilegalmente

19. The IUCN Red List of Threatened Species. 2021. International Union for Conservation of Nature. [Internet]. Disponible: https://www.iucnredlist.org/ search?query=telmatobius\&searchType $=$ species

20. Vellard J. 1951. Estudio sobre batracios andinos. I. El grupo Telmatobius sp. y formas afines. Memorias del Museo de Historia Natural, Lima-Perú

21. Zevallos $S$, Elías RK, Berenguel RA, Weaver TJ, Reading RP. 2016. Batrachochytrium dendrobatidis in Confiscated Telmatobius in Lima, Peru. J Wildlife Dis 52: 949-952. doi: 10.7589/ 2016-01-006 